\title{
Internal femoral component rotation adversely influences load transfer in total knee arthroplasty: a cadaveric navigated study using the Verasense device
}

\author{
William A. Manning ${ }^{1}$ Kanishka M. Ghosh ${ }^{1}$ - Alasdair Blain ${ }^{2} \cdot$ Lee Longstaff $^{1,3}$ • \\ Steven P. Rushton ${ }^{2} \cdot$ David J. Deehan ${ }^{1,4}(\mathbb{C}$
}

Received: 6 January 2017 / Accepted: 7 July 2017 / Published online: 15 July 2017

(c) The Author(s) 2017. This article is an open access publication

\begin{abstract}
Purpose and hypothesis Correct femoral component rotation at knee arthroplasty influences patellar tracking and may determine function at extremes of movement. Additionally, such malrotation may deleteriously influence flexion/extension gap geometry and soft tissue balancing kinematics. Little is known about the effect of subtle rotational change upon load transfer across the tibiofemoral articulation. Our null hypothesis was that femoral component rotation would not influence load across this joint in predictable manner.
\end{abstract}

Methods A cadaveric study was performed to examine load transfer using the orthosensor device, respecting laxity patterns in $6^{\circ}$ of motion, to examine load across the medial and lateral compartments across a full arc of motion. Mixed-effect modelling allowed for quantification of the effect upon load with internal and external femoral component rotation in relation to a datum in a modern singleradius cruciate-retaining primary knee design.

Results No significant change in maximal laxity was found between different femoral rotational states. Internal

David J. Deehan

deehan1@hotmail.co.uk

http://www.nstcsurg.org

1 Newcastle Surgical Training Centre Research

Centre, Freeman Hospital, High Heaton,

Newcastle upon Tyne NE7 7DN, UK

2 School of Biology, University of Newcastle Upon Tyne, Newcastle upon Tyne NE1 7RU, UK

3 Department of Orthopaedics, University Hospital of North Durham, Durham DH1 5TW, UK

4 Institute of Cellular Medicine 4th Floor William Leech Building, Medical School, Newcastle upon Tyne NE2 4HH, UK rotation of the femoral component resulted in significant increase in medial compartment load transfer for knee flexion including and beyond $60^{\circ}$. External rotation of the femoral component within the limits studied did not influence tibiofemoral load transfer.

Conclusions Internal rotation of the femoral component will adversely influence medial compartment load transfer and could lead to premature polyethylene wear on the medial side.

Keywords Femoral rotation $\cdot$ Load $\cdot$ Flexion $\cdot$ Knee arthroplasty

\section{Introduction}

Total knee arthroplasty (TKA) is designed to alleviate pain and restore function with optimal active range of movement. Contemporary primary knee replacement is met with 10 -year survivorship in excess of $95 \%$, up to $20 \%$ of the patient cohort remain less than entirely satisfied and early revision for loss of movement and pain is a significant clinical worry $[1,21,35,41]$. In both registry and singleinstitution series, up to $20 \%$ of the patient group remain dissatisfied with the final outcome with greater than $50 \%$ of such cases related to poor movement or lack of stability and unexplained pain [4]. Modern designs of cruciateretaining knee replacement systems aim to distribute load evenly across the articulating surfaces so as to both reduce polyethylene wear rates and ultimately the TKA revision burden [24]. The kinematic performance of the artificial knee is reliant upon exact placement with respect to the soft tissue envelope of the tibiofemoral and patellofemoral articulations [29]. The common denominator for both is the femoral component. 
Femoral component placement may be performed using either a gap balancing or measured technique [26, 34]. No technique is consistently superior or reliable for femoral component placement [38]. Gap balancing is reliant upon correct tibial resection; otherwise, there is a compound error [14, 22]. Measured resection may introduce a malrotation due to difficulty identifying key anatomical landmarks [25, 43]. Femoral component malrotation will deleteriously influence the geometry of the flexion gap and patellar tracking $[2,17]$. Load and constraint enjoy a complex and not always inverse relationship, and the flexion gap works with the soft tissue envelope and final laxity pattern to determine load across the flexed knee $[8,14]$. Abnormal load may cause pain and stiffness. Our current standard biomechanical assessment is restricted to standing alignment views, and our knowledge of tibiofemoral load transfer in flexion is limited. A greater understanding of the load distribution across the tibiofemoral articulation under defined laxity conditions would allow for the study of the kinematic effect of component rotation and therefore subsequently predict the clinical performance of such a prosthetic joint [20].

In this study, we performed work to quantify the effect of femoral component rotation upon knee laxity and tibiofemoral contact force. Our primary (null) hypothesis was that femoral component rotation would neither influence load transfer across the tibiofemoral articulation nor maximal laxity for the knee arthroplasty construct at key points of knee flexion.

\section{Materials and methods}

\section{Specimen demographics}

This work was performed under formal ethical approval and UK HTA licence within the surgical training facil-

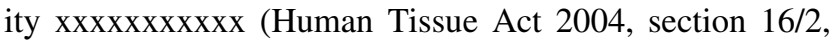
licence number 12148). Whole lower limb cadaveric material without pre-existing radiographic evidence of malrotation/fracture/deformity or arthritic disease was used. Eight fresh frozen lower limbs disarticulated at the hip (three right, five left from Caucasian donors with median BMI 22, range 17-28, male/female ratio 7:1, and mean age 74, range 64-79 years) were prepared in a standardised manner as reported in previous published work [6, $9,14]$. Limbs were mounted onto a custom rig with the tibia hung vertically and secured using set screws to prevent specimen rotation. All muscle groups acting across the knee joint were loaded throughout the experimental procedure using previously validated methodology for the study of cadaveric knee kinetics. In particular, the iliotibial band (ITB), quadriceps muscle group, biceps tendon and medial hamstring apparatus were individually loaded in a physiological manner direction (Fig. 1). Navigation trackers (Stryker eNdtrac Knee Navigation System, Michigan USA) were fixed to the femur and tibia $25 \mathrm{~cm}$ from the joint line, respectively, and in such a manner as to avoid interfering with muscle pull [5]. Previous work had validated the use of eight limbs and confirmed that this provides sufficient power to identify significant differences using this technique with $95 \%$ confidence presuming $80 \%$ power $[8,22]$.

\section{Surgical procedure}

Insertion of a Stryker Triathlon (Michigan USA) singleradius cruciate-retaining TKA (CR-TKA) via a medial parapatellar approach was undertaken using a measured resection technique [27, 34]. The femoral component rotation was determined using the transepicondylar axis, validated using the mid-trochlear axis. A balanced knee was confirmed as that where after minimal soft tissue release, there was passive full extension, full flexion with normal 'no touch' patellar tracking, less than grade 1 laxity for varus valgus stressing at $0^{\circ}, 30^{\circ}, 60^{\circ}$ and $90^{\circ}$ of flexion, firm endpoint to Lachman testing and no laxity for anteroposterior stressing at $90^{\circ}$ of flexion. To achieve such, $8 \mathrm{~mm}$ of distal femoral resection was performed at $5^{\circ}$ valgus intramedullary alignment. Femoral sizing was undertaken using anterior referencing. The mid-point of

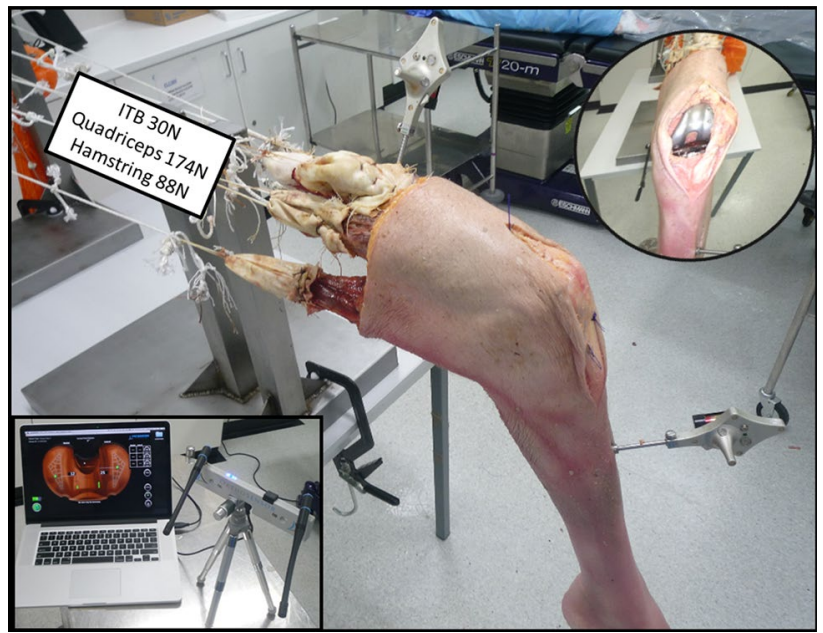

Fig. 1 Experimental setup with custom jig and cadaveric limb loaded using a cable and pulley system to load in physiological directions. ITB $30 \mathrm{~N}$, vastus lateralis $71 \mathrm{~N}$ rectus femoris and vastus intermedialis $61 \mathrm{~N}$, vastus medialis 42 long and short head biceps femoris $44 \mathrm{~N}$, semimembranous and semitendinosus $44 \mathrm{~N}$. An arthrotomy has been performed (inset picture right) showing the Verasense tibial device is in situ with wireless hub connected to display unit (inset picture left). Navigation sensors on tibia and femur track motion and enabling laxity measurements 
the medial epicondyle may be difficult to localise but to reduce any peroperative error both senior authors agreed the mid-point of such so as to define the epicondylar axis. The rotation of the final 4 in 1 femoral cutting block was further validated using the mid-sulcus or Whiteside line. The tibia was then prepared using a 9-mm resection from the superiormost tibial condyle in a plane perpendicular to the anatomical axis with an associated $3^{\circ}$ posterior slope achieved via an extra-medullary jig. The tibial cutting block was centred at the mid-third of the tibial tubercle and formal resection performed. The trial tibial baseplate was centred without restraint on the cut surface. Final orientation of the tibial component was determined after cycling the knee $>20$ times. The final seated position which achieved greatest conformity was marked on the anterior tibial crest using diathermy. For each experiment and each position of femoral component rotation, the tibial component was allowed to find its most conforming position. A standard polyethylene insert was selected and inserted with trial components to ensure the flexion and extension gaps were stable for varus valgus and anteroposterior stressing at the defines points of flexion stated earlier. Full extension was confirmed. The flexion gap allowed for full flexion but was balanced such as to ensure no opening in deep flexion. So as to optimise femoral component stability on the distal femoral resection, lughole screws were inserted and captured the femoral component whilst being buried and not interfering in articulation with the poly-component. This ensured absence of component rotation or glide or translation at any point of the experimental stressing in $6^{\circ}$ of motion during the flexion/extension arc of the experiment (Fig. 2). A Verasense (Orthosensor, Dania FL) of appropriate thickness then replaced the polyethylene insert. With the knee held in $10^{\circ}$ of flexion, the tibial rotation was adjusted and secured when the Verasense system registered a parallel contact point rotation [12]. Medial and lateral tibiofemoral contact forces were recorded as the loaded knee was taken through a range of passive flexion without stressing. No procedure required more than a simple periarticular medial or lateral capsular release to satisfy our criteria for balancing as defined by confirming the load across the medial and lateral compartments were within $15 \mathrm{lbs}$ (pounds-force) of each other, respectively, through a full arc of motion (Fig. 2) [12, 45]. This state defined the datum for femoral component rotation for each knee. To allow for $\pm 3^{\circ}$ of femoral rotation whilst maintaining the original component size, femoral cuts were downsized by $2 \mathrm{~mm}$. The externally rotated (ERF-TKA) and internally rotated (IRF-TKA) states were achieved with the insertion of custom wedges (Fig. 3). The femoral component was fast secured following each rotation using custom cancellous thread lughole screws engaging the stronger subchondral bone. Care was taken to perform surgical closure of the

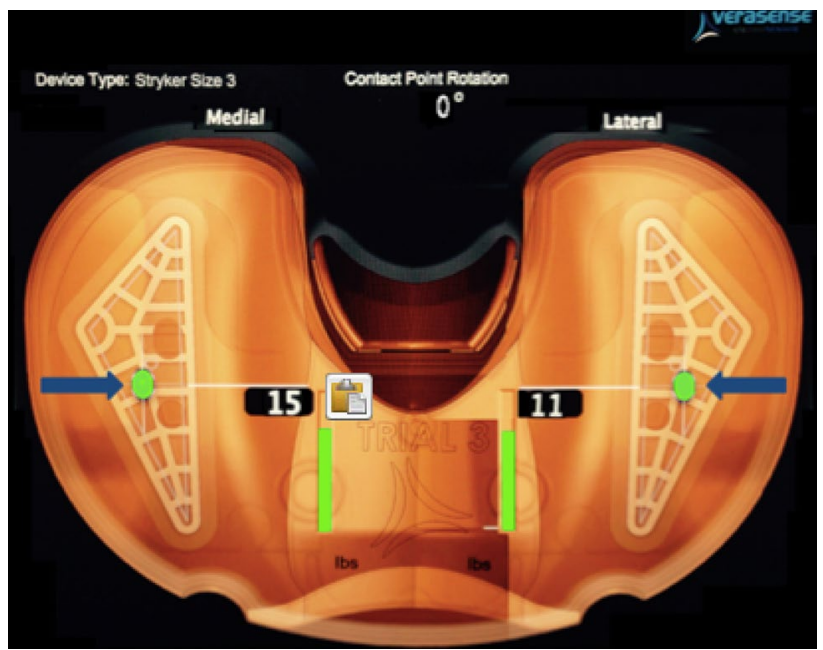

Fig. 2 Verasense display as viewed by the surgeon during balancing. This image shows a rotationally matched tibiofemoral construct as well as a balance soft tissue envelope as defined by medial and lateral compartments contact forces the within $15 \mathrm{lbs}$

arthrotomy via interrupted mattress sutures respecting the anatomy of the parapatellar tissues prior to any testing [31]. The senior authors $(\mathrm{xxx} / \mathrm{xx})$ performed all surgical procedures and stress testing. For all experiments, there were two senior surgeons, a surgical assistant, a manual operator of the orthosensor system, a senior scientist recording the output from the navigation system.

\section{Data capture and analysis}

Data were captured, as per previous validated work, using a standard computerised navigation system with orthosensor provided range of compatible tibial trials [14, 37]. Knees were manually stressed to mimic intraoperative laxity assessment. A datum was taken from the knee in extension from which maximal displacements of the tibia in relation to the fixed femur were tracked via computer navigation (Stryker eNdtrac Knee Navigation System, Michigan, USA) to an accuracy of $\pm 0.5 \mathrm{~mm}$ in $6^{\circ}$ of freedom [5, 10, 28]. For each TKA condition, maximal displacements (anteroposterior, varus, valgus, internal and external rotation) were each recorded at five angles of flexion $\left(0^{\circ}, 30^{\circ}, 60^{\circ}, 90^{\circ}\right.$ and $110^{\circ}$ ). To reduce hysteresis, repeated flexion cycles were undertaken between measurements and ensured compartment forces remained constant during passive flexion. After each set of measurements, the output instrumentation was reset at zero. The Verasense device recorded tibiofemoral contact force (lbs/force) and contact points continuously during testing (millimetre accuracy $\pm 2 \mathrm{~mm}-\mathrm{C}$. Anderson, OrthoSensor, personal communication 03.08.2015) with additional data capture under maximal stress at each of the five angles of flexion. Three knee conditions were defined 


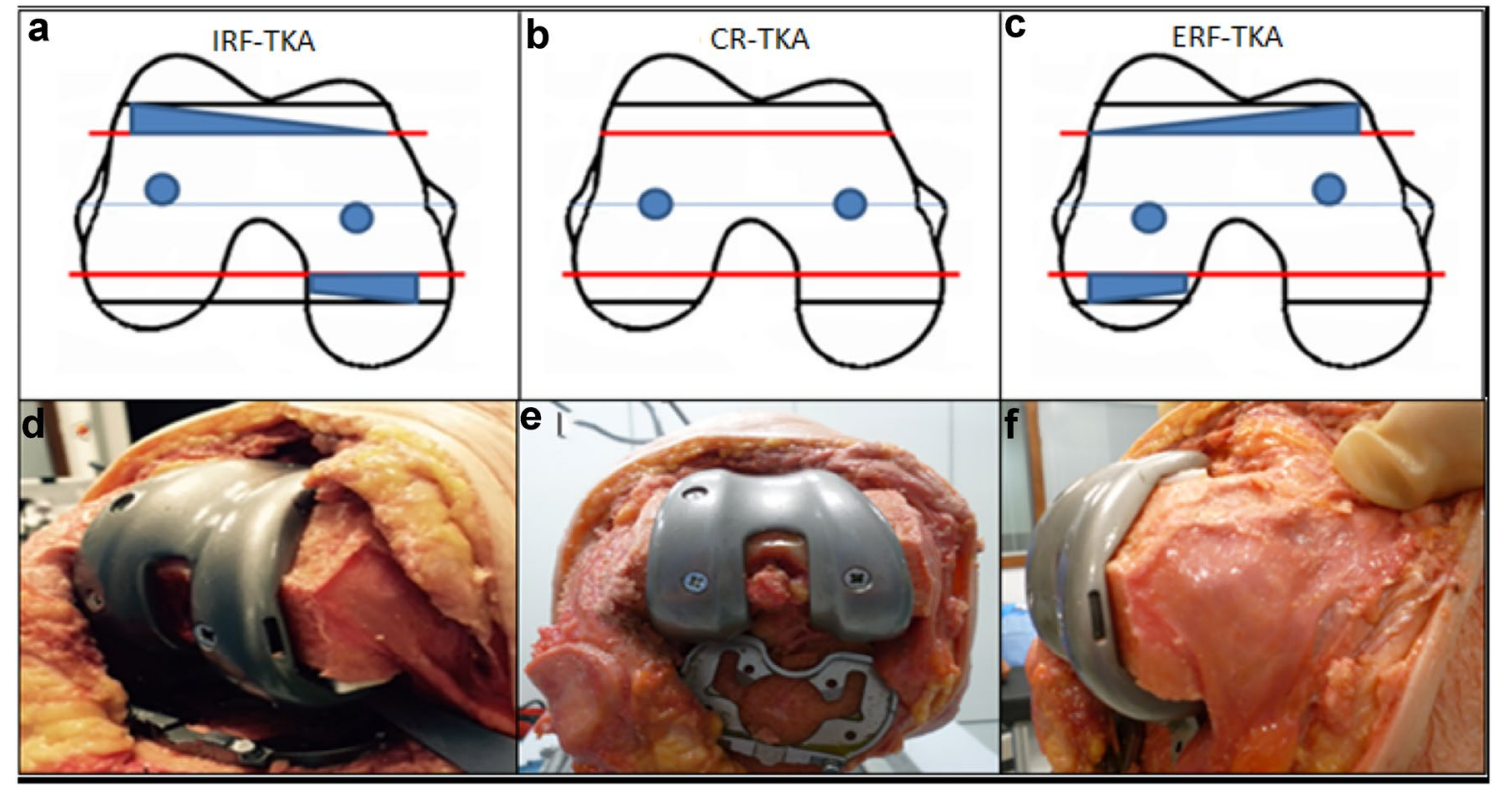

Fig. 3 Pictorial representation of femoral component alignment, exaggerated for demonstration, showing the neutrally aligned CRTKA (b), and the rotated femoral component to create the IRF-TKA (a) and ERF-TKA (c). Black lines demonstrating original femoral siz-

with the internal and external rotatory states compared with the neutral or datum. These were the internally rotated femoral component (IRF-TKA) and the externally rotated femoral component (ERF-TKA) (Fig. 3).

\section{Statistical analysis}

Mixed-effect modelling was used to quantify the effect of flexion angle, direction of movement and implantation of TKA upon laxity [30, 32]. Displacements were used as the response variable, with TKA and flexion as covariates. Student's $t$ test was used to compare differences in tibiofemoral force and contact point measurements. Significance was set at a level of $p<0.05$.

\section{Results}

\section{Native knee}

Total tibial rotation (combined internal and external rotation for each point of knee flexion of replaced state) and maximal varus/valgus laxity were found to increase consistently from full extension to deep flexion for all knee specimens. Rotational and coronal laxity did also increase with knee flexion within the native knee. This increase in laxity/rotation comparing the native versus the replaced state was significant for maximal anteroposterior movement (AD) when comparing $110^{\circ}-30^{\circ}$ (Fig. 4a). ing and red lines demonstrating the downsized femoral cuts ( $2 \mathrm{~mm})$ to accommodate for component rotation. Custom polyethylene inserts d, $\mathbf{f}$ provide reproducible rotation whilst maintaining correct femoral size and implant stability aided by lughole screws $\mathbf{e}$ for stress testing

\section{CR-TKA laxity pattern}

A decrease in laxity was found for all knees after implantation of a single-radius (CR-TKA) cruciate-retaining knee design when compared to the native state. This reduction in maximal laxity only reached significance for rotatory laxity in higher levels of knee flexion beyond $90^{\circ}$ (Fig. $4 \mathrm{a}-\mathrm{c}$ ).

\section{Load transfer}

Assessment of the unstressed flexion arc showed that contact forces across the medial and lateral compartments showed no statistical difference at each angle of flexion in the CR-TKA (Fig. 5a). Tibiofemoral contact force demonstrated equilibrium of load in the primary CR-TKA at each angle of flexion. The total force across the tibiofemoral joint was seen to decrease with flexion. An inverse relationship for total contact load versus maximal laxity was, as expected, found consistently for each knee.

\section{IRF-TKA laxity pattern}

Maximal anteroposterior was significantly increased for the internally rotated femoral component state beyond $60^{\circ}$ when compared to both the neutrally and externally rotated femoral component states. Varus valgus maximal movement and maximal rotatory motion assessment failed to 

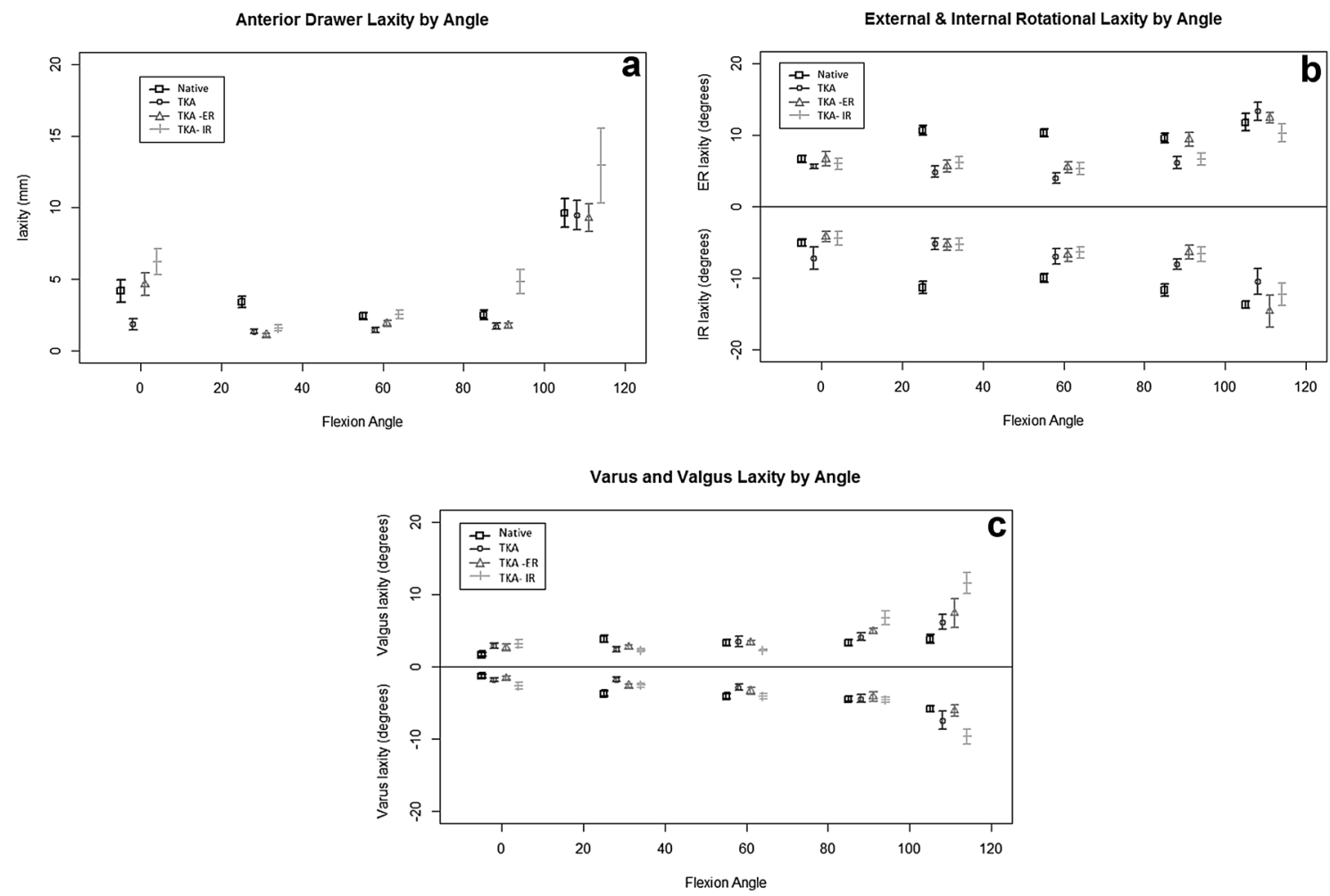

Fig. 4 a Maximal anterior laxity (mm) under anterior draw stress testing with knee flexion (degrees). Native knee (square), CR-TKA (circle), ERF-TKA (triangle), IRF-TKA (solid line) $n=8$. b Maximal rotational laxity (degrees) with knee flexion (degrees). Native knee

identify any difference for the internally rotated component state when compared to neutral or external rotation (Fig. 4a-c).

\section{Load transfer}

Load across the medial compartment was significantly increased at and beyond $60^{\circ}$ of flexion with the internal femoral component state (Fig. 5b). This was most marked when stressing the knee with maximal internal rotation beyond $60^{\circ}$ of flexion. At $90^{\circ}$ flexion, a mean of $83 \%$ of the total contact load was borne by the medial compartment in the ERF-TKA compared to 47 and $43 \%$ in the CR-TKA and ERF-TKA, respectively $(p<0.05)$.

\section{ERF-TKA laxity pattern}

The laxity pattern for the externally rotated femoral component knee arthroplasty (ERF-TKA) did not significantly alter when compared to the primary neutral position of the femoral component. Whilst the greatest difference between the ERF-TKA and CR-TKA was found at $110^{\circ}$ with an increase in rotational laxity, this failed to reach statistical significance. No significant difference at any angle of (square), CR-TKA (circle), ERF-TKA (triangle), IRF-TKA (solid line) $n=8$. c Maximal varus valgus laxity (degrees) with knee flexion (degrees). Native knee (square), CR-TKA (circle), ERF-TKA (triangle), IRF-TKA (solid line) $n=8$

flexion was found between the neutrally and externally rotated femoral component arthroplasty states for maximal anteroposterior movement or varus valgus stressing (Fig. 4a-c).

\section{Load transfer}

Similar load was found across the medial and lateral femoral compartments between $0^{\circ}$ and $30^{\circ}$ of flexion when comparing the ERF-TKA versus CR-TKA states. Between $60^{\circ}$ and $110^{\circ}$ load increased across the lateral compartment under rotational stress for the ER-TKA when compared to the CR-TKA. This change did not, however, reach significance (Fig. 5c). It was representative of a proportional increase in lateral compartment loading in the ER-TKA state compared to the CR-TKA in mid to deep flexion. Overall, the ERF-TKA load pattern very closely resembled the pattern of contact force recorded for the neutrally aligned femoral component (CR-TKA) state.

\section{Load transfer with varus/valgus stress testing}

There was consistency of load transfer across the medial and lateral compartments for both neutral datum femoral 
Flexion Arc Sensor Recordings

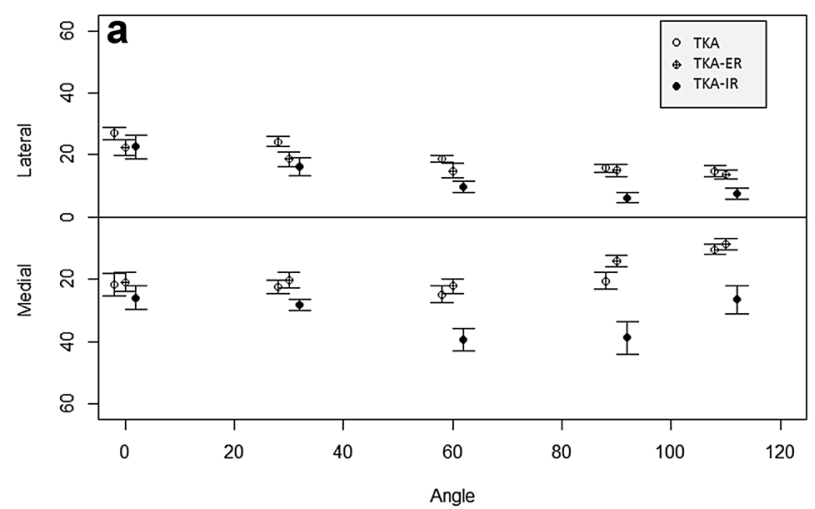

ER Sensor Recordings

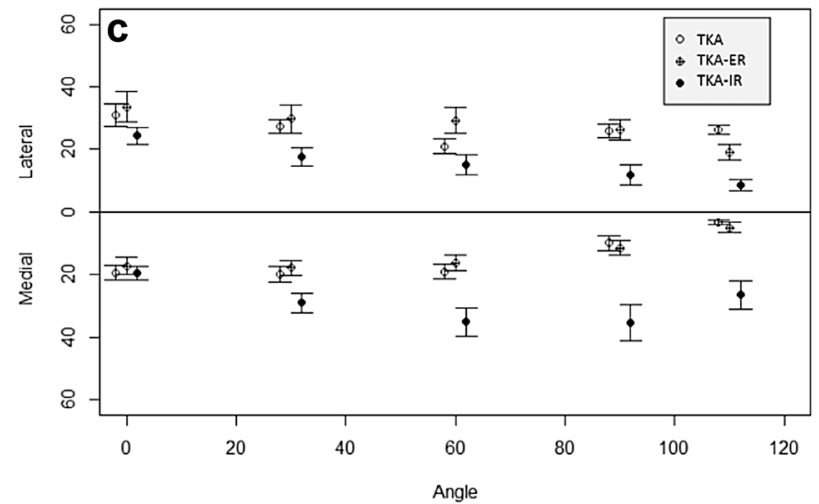

IR Sensor Recordings

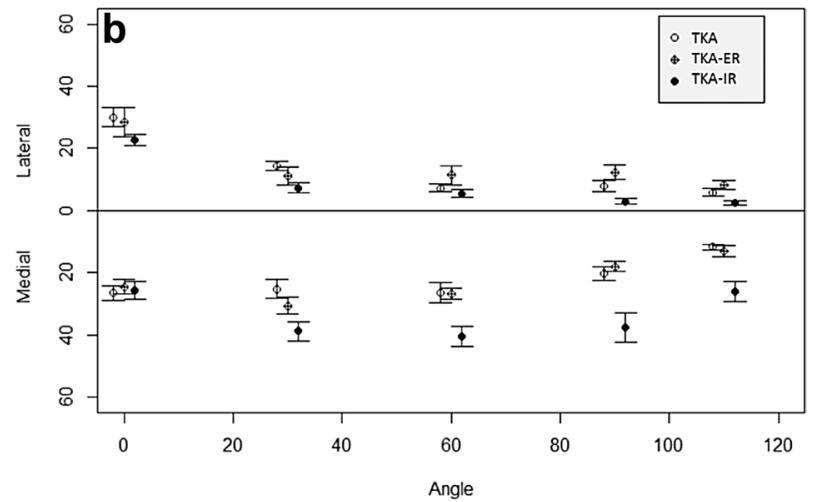

Valgus Sensor Recordings

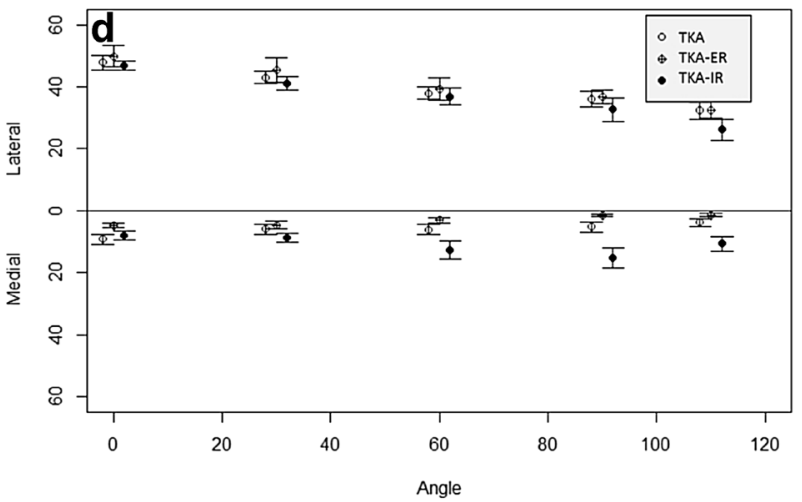

Varus Sensor Recordings

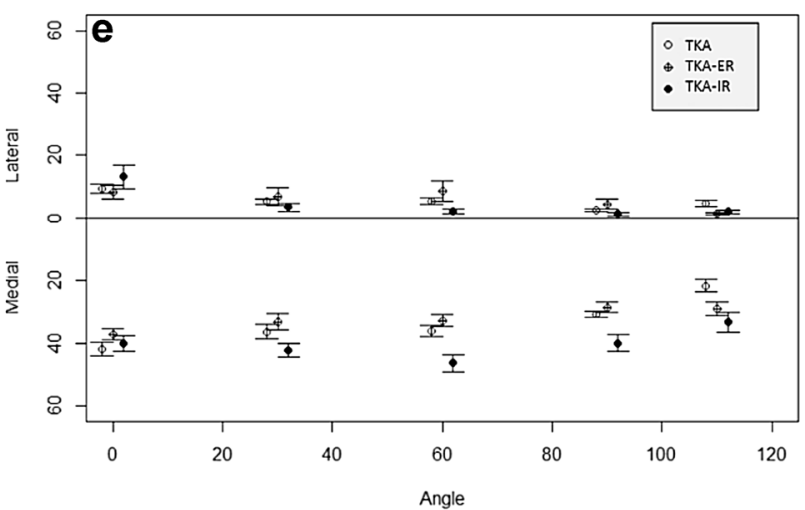

Fig. 5 a Mean tibiofemoral contact force (lbs) during a normal flexion arc (degrees) with no external stress applied. CR-TKA (clear circle left), ERF-TKA (crossed circle middle), IRF-TKA (solid circle right) $n=8$. b Mean tibiofemoral contact force (lbs) at maximal internal rotation stress with knee flexion (degrees). CR-TKA (clear circle left), ERF-TKA (crossed circle middle), IRF-TKA (solid circle right) $n=8$. c Mean tibiofemoral contact force (lbs) at maximal external rotation stress with knee flexion (degrees). CR-TKA (clear circle left), ERF-TKA (crossed circle middle), IRF-TKA (solid circle right) $n=8$. d Mean tibiofemoral contact force (lbs) at maximal valgus stress with knee flexion (degrees). CR-TKA (clear circle left), ERF-TKA (crossed circle middle), IRF-TKA (solid circle right) $n=8$. e Mean tibiofemoral contact force (lbs) at maximal varus stress with knee flexion (degrees). CR-TKA (clear circle left), ERF-TKA (crossed circle middle), IRF-TKA (solid circle right) $n=8$

significance, there was a consistent increase in load transfer on the medial compartment in higher degrees of knee flexion for both varus and valgus load (Fig. 5d, e). 


\section{Discussion}

The principal findings of this work were the rejection of the null hypothesis that femoral component rotation would not influence load transfer across the tibiofemoral joint. Internal rotation of the femoral component led to increased load across the medial tibiofemoral compartment most markedly beyond mid-flexion. This redistribution of load was found even with a neutrally aligned knee replacement. It is possible that such increased load across the medial compartment could explain the pain reported by patients in clinical series with malrotated components [3, 17, 29]. The study failed to demonstrate a reciprocal increase in load on the lateral side with external rotation of the femoral component. Further, whilst the relationship between laxity patterns for total load across the tibiofemoral articulation mimicked those previous reported, the experiment failed to demonstrate a significant inverse relationship of such at the extremes of motion. Subtle internal malrotation of the femoral component resulted in a substantial increase in load transfer across the medial compartment. These differences were most evident for both varus and valgus stress testing in flexion. This dynamic biomechanical imbalance in flexion would not be detected in any standard long leg film view performed postoperatively and sheds light on the limitations of static views to determine biomechanical axes after surgery.

There are weaknesses inherent in the use of cadaveric material for such experimental work. None of the knees used in this work exhibited arthritic change or deformity. And whilst this does minimise the confounding effects of soft tissue contracture or bone loss, it may not entirely accurately replicate the operative state. However, the use of non-arthritic knees did allow for better accuracy when determining anatomical landmarks such as the epicondylar axis and ensured that baseline implantation was simple reliable and achieved quickly by the two senior operating surgeons. In contrast to previous work, all muscle groups acting across the knee joint were loaded as per previous methodology [22]. Particular attention was made to ensure free full tibiofemoral and patellar movement in the native loaded state and after primary implantation, cognisant of previous work, thereby confirming the importance of load across the joint to achievement of inherent stability [16, $22,46]$. The loads used in this study were subphysiological consistent with previous work so as to reduce risk of muscle tearing. Time zero assessment takes no account of the patient size, demographics or activity level. However, the use of cadaveric knees does allow for repeated-measures statistical analyses and reduces the confounding influence of pathological or functional differences between patients, thereby potentially enhancing the power of this work. Furthermore, prior to experimentation, all limbs had undergone radiographic assessment to exclude peri-articular deformity, degenerative change and confirmed neutral frontal plane alignment, which was confirmed as neutral for all limbs.

Excess internal femoral rotation in the absence of tibial malrotation is reported as an indication for revision arthroplasty [13, 23]. Several series confirm that revision for femoral component malrotation in isolation will achieve improved function with particular emphasis on functional measures which rely upon a stable and balanced mid-flexion tibiofemoral articulation [13, 23, 42]. Unlike the work of Thompson et al., we did allow for the tibia to sit on the tibial surface where there was maximal conformity, by cycling the knee after fixing the femoral component and allowing the tibia to sit accordingly, congruity was optimised from a load perspective [7, 33, 40]. Internal femoral component rotation with overstuffing of the medial compartment in flexion may stretch the MCL leading to pain, stiffness, failure to facilitate safe flexion and impaired varus/valgus laxity [11, 22, 33, 40]. Increased compartmental load on the medial side may lead to premature failure and suboptimal performance due to abnormal non-physiological load being shared across the articulation. Jeffcote et al. [15] correlated tibiofemoral forces with collateral ligament strain when increasing the flexion gap. Medial tibial pain has been reported in this patient cohort, and the finding of increased medial compartmental load with internal femoral component rotation could offer an explanation for such. We believe that this is the first work to identify abnormal preferential asymmetrical loading of the medial compartment without evidence of a reciprocal effect on laxity. Much previous work has argued that achievement of a quadrilateral flexion gap and the use of ligament tension devices may aid with load distribution but without determining load per se. However, this work does raise concerns about how reliable peroperative manual stress is at determining equivalent loading across the medial and lateral compartments [44]. Previous CT work has found rotational alignment errors in vivo to range from $13^{\circ}$ of internal rotation to as much as $16^{\circ}$ of external rotation have been documented [36]. Correct coronal plane alignment is associated with good clinical outcomes, but little is known of the correct mechanical axis in flexion [19]. Our work has focused on a much narrower range of rotation and despite such has still identified significant variation in load transfer. Greater errors in 3D placement can underscore the early clinical failures with patients reporting suboptimal functional performance from mediolateral pathological laxity and inevitable pain from loosening and abnormal loading of the subchondral bone [18].

Our work failed to find differences in load on the lateral side with external rotation of the femoral component. It is known that the posterolateral corner, additionally, is ineffective in flexion [39]. This apparent capacity to dissipate 
load in flexion could explain the minimal change in load on the lateral side with excess femoral external rotation. Recent work on the kinematics of femoral component rotation did examine the impact of femoral and tibial component rotation upon load in the surrounding muscle groups [40]. These workers found that femoral component rotation principally influenced load in the quadriceps apparatus and collateral ligaments, thereby determining varus valgus movement. However, no work was done to examine load across the joint. Our work has added to this knowledge by relating in isolation femoral component rotation, albeit with more subtle rotational margins [40].

\section{Compliance with ethical standards}

Conflict of interest There are no conflicts of interest as per previous cadaveric work.

Funding Funding was provided by Newcastle Charitable Trustees and Stryker Research Fund Europe.

Ethical approval As per HTA licence copied to Newcastle Hospital / Newcastle University for the nstc http://www.nstcsurg.org.uk, ethical approval is institutional.

Informed consent No informed consent was required as no patient material was used and no patient was involved in this work.

Open Access This article is distributed under the terms of the Creative Commons Attribution 4.0 International License (http:// creativecommons.org/licenses/by/4.0/), which permits unrestricted use, distribution, and reproduction in any medium, provided you give appropriate credit to the original author(s) and the source, provide a link to the Creative Commons license, and indicate if changes were made.

\section{References}

1. Baker PN, Rushton S, Jameson SS, Reed M, Gregg P, Deehan DJ (2013) Patient satisfaction with total knee replacement cannot be predicted from pre-operative variables alone: a cohort study from the National Joint Registry for England and Wales. Bone Joint J 95B(10):1359-1365

2. Berger RA, Rubash HE, Seel NJ, Thompson WH, Crossett LS (1993) Determining the rotational alignment of the femoral component in total knee arthroplasty using the epicondylar axis. Clin Orthop Relat Res 286:40-47

3. Berger RA, Crossett LS, Jacobs JJ, Rubash HE (1998) Malrotation causing patellofemoral complications after total knee arthroplasty. Clin Orthop Relat Res 356:144-153

4. Dennis DA (2004) Evaluation of painful total knee arthroplasty. J Arthroplasty 19(4 Suppl 1):35-40

5. Elfring R, De la Fuebte M, Radermacher K (2010) Assessment of optical localizer accuracy for computer aided surgery systems. Comput Aided Surg 15:1-12

6. Farahmand F, Senavongse W, Amis AA (1998) Quantitative study of the quadriceps muscles and trochlear groove geometry related to instability of the patellofemoral joint. J Orthop Res $16: 136-143$
7. Feczko PZ, Pijls BG, van Steijn MJ, van Rhijn LW, Arts JJ, Emans PJ (2016) Tibial component rotation in total knee arthroplasty. BMC Musculoskelet Disord 16(17):87

8. Ghosh KM, Blain AP, Longstaff L, Blain AP, Longstaff L, Rushton S, Amis AA, Deehan DJ (2014) Can we define envelope of laxity during navigated knee arthroplasty? Knee Surg Sports Traumatol Arthrosc 22:1736-1743

9. Ghosh KM, Hunt N, Blain AP, Hunt N, Blain A, Athwal KK, Longstaff L, Amis AA, Rushton S, Deehan DJ (2015) Isolated popliteus tendon injury does not lead to abnormal laxity in posterior-stabilised total knee arthroplasty. Knee Surg Sports Traumatol Arthrosc 23(6):1763-1769

10. Grood ES, Suntay WJ (1983) A joint coordinate system for the clinical description of three-dimensional motions: application to the knee. J Biomech Eng 105:136-144

11. Gu Y, Howell SM, Hull ML (2016) Simulation of total knee arthroplasty in $5^{\circ}$ or $7^{\circ}$ valgus: a study of gap imbalances and changes in limb and knee alignments from native. J Orthop Res. doi:10.1002/jor.23492

12. Gustke KA, Golladay GJ, Roche MW, Elson LC, Anderson CR (2014) A new method for defining balance: promising shortterm clinical outcomes of sensor-guided TKA. J Arthroplasty 29:955-960

13. Hofmann S, Seitlinger G, Djahani O, Pietsch M (2011) The painful knee after TKA: a diagnostic algorithm for failure analysis. Knee Surg Sports Traumatol Arthrosc 19(9):1442-1452

14. Hunt NC, Ghosh KM, Blain AP, Blain AP, Athwal KK, Rushton SP, Amis AA, Longstaff LM, Deehan DJ (2014) How does laxity after single radius total knee arthroplasty compare with the native knee? J Orthop Res 32:1208-1213

15. Jeffcote B, Nicholls R, Schirm A, Kuster MS (2007) The variation in medial and lateral collateral ligament strain and tibiofemoral forces following changes in the flexion and extension gaps in total knee replacement. A laboratory experiment using cadaver knees. J Bone Joint Surg Br 89(11):1528-1533

16. Kwak SD, Ahmad CS, Gardner TR, Grelsamer RP, Henry JH, Blankevoort L, Ateshian G, Mow VC (2000) Hamstrings and iliotibial band forces affect knee kinematics and contact pattern. J Orth Res 18:101-118

17. Lakstein D, Zarrabian M, Kosashvili Y, Safir O, Gross AE, Backstein D (2010) Revision total knee arthroplasty for component malrotation is highly beneficial: a case control study. J Arthroplasty 25:1047-1052

18. Le DH, Goodman SB, Maloney WJ, Huddleston JI (2014) Current modes of failure in TKA: infection, instability, and stiffness predominate. Clin Orthop Relat Res 47:2197-2200

19. Longstaff LM, Sloan K, Stamp N, Scaddan M, Beaver R (2009) Good alignment after total knee arthroplasty leads to faster rehabilitation and better function. J Arthroplasty 24(4):570-578

20. Malkani AL, Hitt KD, Badarudeen S, Lewis C, Cherian J, Elmallah R, Mont MA (2016) The difficult primary total knee arthroplasty. Instr Course Lect 65:243-265

21. Mannion AF, Kämpfen S, Munzinger U, Kramers-de Quervain I (2009) The role of patient expectations in predicting outcome after total knee arthroplasty. Arthritis Res Ther 11(5):R139

22. Merican AM, Ghosh KM, Iranpour F, Deehan DJ, Amis AA (2011) The effect of femoral component rotation on the kinematics of the tibiofemoral and patellofemoral joints after total knee arthroplasty. Knee Surg Sports Traumatol Arthrosc 19(9):1479-1487

23. Miller MC, Berger RA, Petrella AJ, Karmas A, Rubash HE (2001) Optimizing femoral component rotation in total knee arthroplasty. Clin Orthop Relat Res 392:38-45

24. Nedopil AJ, Howell SM, Hull ML (2016) Does malrotation of the tibial and femoral components compromise function in 
kinematically aligned total knee arthroplasty? Orthop Clin North Am 47(1):41-50

25. Nikolaides AP, Kenanidis EI, Papavasiliou KA, Sayegh FE, Tsitouridis I, Kapetanos GA (2014) Measured resection versus gap balancing technique for femoral rotational alignment: a prospective study. J Orthop Surg 22:158-162

26. Olcott CW, Scott RD (2000) A comparison of 4 intraoperative methods to determine femoral component rotation during total knee arthroplasty. J Arthroplasty 15(1):22-26

27. Oussedik S, Scholes C, Ferguson D, Roe J, Parker D (2012) Is femoral component rotation in a TKA reliably guided by the functional flexion axis? Clin Orthop Relat Res 470:3227-3232

28. Pearle AD, Solomon DJ, Wanich T, Moreau-Gaudry A, Granchi CC, Wickiewicz TL, Warren RF (2007) Reliability of navigated knee stability examination: a cadaveric evaluation. Am J Sports Med 35:1315-1320

29. Pietsch M, Hofmann S (2012) Early revision for isolated internal malrotation of the femoral component in total knee arthroplasty. Knee Surg Sports Traumatol Arthrosc 20(6):1057-1063

30. Pinheiro JC, Bates DM (2000) Fitting linear mixed-effects models. In: Mixed-effects models in S and S-PLUS. Springer, New York, pp 133-139

31. Plate JF, Seyler TM, Halvorson JJ, Santago AC II, Lang JE (2014) Non-anatomical capsular closure of a standard parapatellar knee arthrotomy leads to patellar maltracking and decreased range of motion: a cadaver study. Knee Surg Sports Traumatol Arthrosc 22(3):543-549

32. Ranstam J (2012) Repeated measurements, bilateral observations and pseudoreplicates, why does it matter? Osteoarthr Cartil 20(6):473-475

33. Scott RD (2006) Total knee arthroplasty. Elsevier, Philadelphia, pp 63-68

34. Scott RD (2013) Femoral and tibial component rotation in total knee arthroplasty: methods and consequences. Bone Joint $\mathrm{J}$ 95B(11SupplA): 140-143

35. Scott CEH, Howie CR, MacDonald D, Biant LC (2010) Predicting dissatisfaction following total knee replacement: a prospective study of 1217 patients. J Bone Joint Surg Br 92B:1253-1258
36. Siston RA, Patel JJ, Goodman SB, Delp SL, Giori NJ (2005) The variability of femoral rotational alignment in total knee arthroplasty. J Bone Joint Surg Am 87:2276-2280

37. Stulberg SD, Loan P, Sarin V (2002) Computer-assisted navigation in total knee replacement: results of an initial experience in thirty-five patients. J Bone Joint Surg Am 84A(Suppl 2):90-98

38. Talbot S, Bartlett $\mathbf{J}$ (2008) The anterior surface of the femur as a new landmark for femoral component rotation in total knee arthroplasty. Knee Surg Sports Traumatol Arthrosc 16(3):258-262

39. Thaunat M, Pioger C, Chatellard R, Conteduca J, Khaleel A, Sonnery-Cottet B (2014) The arcuate ligament revisited: role of the posterolateral structures in providing static stability in the knee joint. Knee Surg Sports Traumatol Arthrosc 22:2121-2127

40. Thompson JA, Hast MW, Granger JF, Piazza SJ, Siston RA (2011) Biomechanical effects of total knee arthroplasty component malrotation: a computational simulation. J Orthop Res 29(7):969-975

41. Toms AD, Mandalia V, Haigh R, Hopwood B (2009) The management of patients with painful total knee replacement. J Bone Joint Surg Br 91(2):143-150

42. Valkering KP, Breugem SJ, van den Bekerom MP, Tuinebreijer WE, van Geenen RC (2015) Effect of rotational alignment on outcome of total knee arthroplasty. Acta Orthop 86(4):432-439

43. Vince K (2016) Mid-flexion instability after total knee arthroplasty: woolly thinking or a real concern? Bone Joint J $98 \mathrm{~B}(1$ Suppl A):84-88

44. Walde TA, Bussert J, Sehmisch S, Balcarek P, Stürmer KM, Walde HJ, Frosch KH (2010) Optimized functional femoral rotation in navigated total knee arthroplasty considering ligament tension. Knee 17(6):381-386

45. Walker PS, Meere PA, Bell CP (2014) Effects of surgical variables in balancing of total knee replacements using an instrumented tibial trial. Knee 21(1):156-161

46. Worland RL, Jessup DE, Johnson GVV, Alemparte JA, Tanaka S, Rex FS, Keenan J (2002) The effect of femoral component rotation and asymmetry in total knee replacements. Orthopedics $25: 1045-1048$ 